\title{
An unusual presentation of prominent crista terminalis mimicking a right atrial mass: a case report
}

\author{
Jiang Wang ${ }^{1 \dagger}$, Gang Wang ${ }^{2}$, Xiao Bi ${ }^{3}$, Ran Zhang ${ }^{4}$ and Changfu Liu ${ }^{4 * \dagger}$ (D)
}

\begin{abstract}
Background: The crista terminalis is a variation of normal anatomical structure within the right atrium which may be misdiagnosed with an abnormal atrial mass normally visualized in the standard views on the transthoracic echocardiogram.

Case presentation: In this case presentation, we demonstrated a rare case report describing the accidental discovery of a right atrial mass-like structure in a 54-year old Asian man without physical discomfort during an echocardiographic examination. These findings naturally caused some concern as the differential diagnosis such as right atrial myxoma or thrombus and further examination were organized. The subsequent positron emission tomography/magnetic resonance imaging (PET/MRI) differentiated a true right atrial mass from a strip extending into the atrium in accordance with prominent crista terminalis.

Conclusion: A preferable understanding of the complex anatomy and PET/MRI appearance of a prominent crista terminalis will minimize the misdiagnosis of this structure and avoiding unnecessary anxiety and more invasive examinations.
\end{abstract}

Keywords: Crista terminalis, Right atrial mass, PET/MRI, Echocardiogram

\section{Background}

A prominent crista terminalis is a well-defined fibromuscular ridge formed by the junction of the sinus venosus and primitive right atrium (RA) extending along the posterolateral aspect of the right atrial wall, which is a normal anatomic variant and recognized by echocardiography occasionally $[1,2]$. However, this variant structure can be misdiagnosed with true right atrial mass like tumor, thrombus or vegetation [3-11]. Compared with the method in echocardiography, the ability of cardiac positron emission tomography/magnetic resonance imaging (PET/MRI) to tissue characteristics enables differentiation between tumor, thrombus, structural abnormalities and normal variant anatomical structures, which is frequently recommended for further evaluation in presence of an unclear cardiac mass and minimizes the misdiagnosis of

\footnotetext{
* Correspondence: liucf301@126.com

† Jiang Wang and Changfu Liu contributed equally to this work.

${ }^{4}$ Department of Cardiology, General Hospital of Chinese People's Liberation

Army, 28th Fuxing Road, Beijing 100853, China

Full list of author information is available at the end of the article
}

variant structure [12-14]. In this case report, we present a case of a cardial mass within right atrium on echocardiography, which is differentiated from a pathological cardiac mass by multiplanar imaging of cardiac MRI, enhancement and ${ }^{18}$ F-FDG uptake of tissue for understanding of the complex anatomy of the heart and its anatomical variations. An appreciation of the anatomy and MRI appearance of the crista terminalis will minimize the misdiagnosis of this structure and avoid additional unnecessary and more invasive tests.

\section{Case presentation}

A 54-year-old man was admitted to our hospital for a mass in RA on an echocardiography examination occasionally. There was no symptom or sign of fever, chest pain, dizziness, palpitations and no history of a heart disease and tumor. He was a teacher, running and training on a weekly basis. He also was a non-smoker and drank $50 \mathrm{ml}$ Chinese liquor each day for 30 years. There was no other medical history of note and no family history of sudden death. Clinically, the patient has stable vital 
signs with no fever, a heart rate of 68 beats per minute and blood pressure of $128 / 79 \mathrm{mmHg}$. The serum tumor markers and D-dimer were normal. An electrocardiogram (ECG) showed normal sinus rhythm. The chest $\mathrm{X}$-ray showed normal cardiac size and clear lungs.

The echocardiography (Siemens, ACUSON SC2000) showed normal left ventricular systolic function (ejection fraction 66\%), normal left and right ventricular cavities and normal cardiac valves. Only mild tricuspid and aortic valve regurgitate. Incidentally, an apparently smooth mass-like echogenic structure $\left(9^{*} 11 \mathrm{~mm}\right)$ attached to tricuspid valve was noted in the right atrium and suggestive of a thrombus or a tumor in four-chamber apical view (Fig. 1).

Due to the limitation of acoustic window of echocardiography, the patient was arranged to our center of nuclear medicine for PET/MRI for diagnostic work up of the mass to be determined. Cardiac PET/MRI was performed with Siemens Biograph mMR (Software version B20P, Siemens Healthcare, Erlangen, Germany). The MRI showed the findings: Cine gradient-echo image in four-chamber view confirmed the presence of a banded structure (arrowhead) attached to the posterior wall of RA and the mass moved during systole and diastole period (Fig. 2c) (See Additional file 1: Video S1). On T2-weighted short tau inversion recovery (T2-STIR) of the four chamber and short axis view (Figs. 2a and 3a), the mass (arrowhead) had similar signal intensity to myocardium revealing a myocardial structure. On T2-STIR with fat suppression of the four chamber and short axis view (Figs. 2b and $3 \mathrm{~b}$ ), the results were consistent with T2-STIR images. There was no abnormal enhancement about the mass (arrowhead) on late gadolinium enhancement (LGE)-MRI (Figs. 2d and 3c).
Furthermore, PET/MRI indicated no obvious ${ }^{18} \mathrm{~F}$-fluorodeoxyglucose $\left({ }^{18} \mathrm{~F}\right.$-FDG) uptake on the mass (Fig. 4, arrowhead), which exclude malignant tumor. These typical findings detected by PET/MRI lead to the diagnosis of a prominent crista terminalis. The case follow-up hitherto had no special symptom.

\section{Discussion}

The crista terminalis is a landmark of the RA, extending along the posterolateral aspect of the right atrial wall. It is the fibromuscular ridge begins at the upper portion of the septal surface and passes anteriorly to the opening of the superior vena cava and terminates at the lateral side of the entrance of the inferior vena cava. When the embryo developed to sixth weeks, the right angle of the venous sinus, the superior inferior vena cava and the original atrium were fused to form the crista terminalis. It divided the RA into two sections: atrium proper and sinus venarum cavarum and corresponded to the sulcus terminalis of the surface of right atrium $[15,16]$. On microscopic view, the crista terminalis consists of three cells: working cells, pacemaker cells and transitional cell, of which the myofibers consist of two layers; the deep is thick and the superficial is only in the middle to lower part of crista terminalis. The pacemaker cells play no role in sinus pacemaking and conduction. While in the majority of patients, the crista terminalis is a normal and benign mass. The changes of velocity and direction of conduction result in cardiac arrhythmia on pathophysiological condition and the anatomic importance of crista terminalis related to arrhythmia is well documented, which has been studied most extensively by electrophysiologists. The crista terminalis can initiate ectopic atrial beats, especially right atrial tachyarrhythmia, which lead

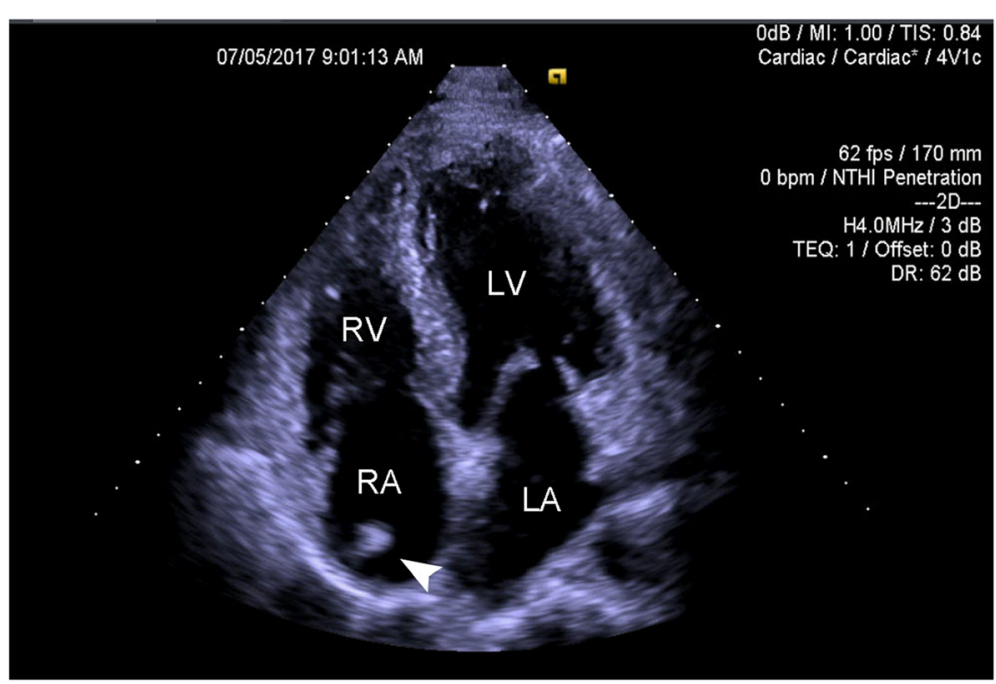

Fig. 1 Transthoracic echocardiogram, in four-chamber apical view, showed an echogenic mass (arrowhead) in the right atrium. LA: left atrium, LV: left ventricle, RA: right atrium, RV: right ventricle 

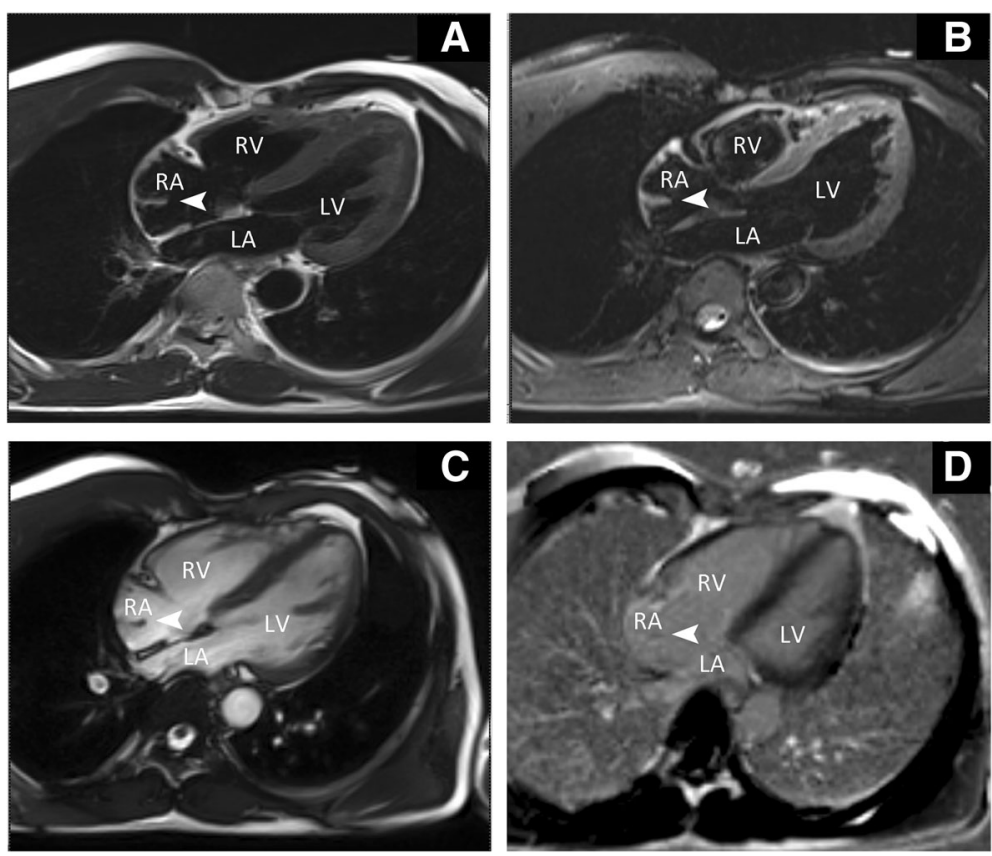

Fig. 2 Four chamber view. a. On T2-STIR image, the mass (arrowhead) had similar signal intensity to myocardium revealing a myocardial structure and excluding lipoma. b. On T2-STIR with fat suppression image, the consequence is consistent with (a). c. The cine MRI confirmed a mass (arrow-head) attached to the posterior wall of the right atrium. D. LGE-sequences indicated no abnormal enhancement of the mass (arrow-head). T2-STIR, T2-weighted short tau inversion recovery; LA, left atrium; LV, left ventricle; RA, right atrium; RV, right ventricle; LGE, late gadolinium enhancement

to paroxysmal atrial fibrillation or atrial flutter [16]. Sanchez-Quintana et al. [17] found that two-thirds of focal right atrial tachycardia, in the absence of structure heart disease, arise from the crista terminalis. In our case report, the patient has no clinical symptoms and the ECG is normal. We can recommend him review regularly and visit periodically.

The differential diagnosis of the crista terminalis is particularly important, especially the mass in the RA, which is mostly detected by echocardiography except for the clinical manifestations of primary diseases. The tumor, thrombus and vegetation are the common cardial mass. And the tumor according to source are divided into primary tumors and metastases $[18,19]$. So the early detection and accurate identification of these mass are of great significance in the diagnosis and prognosis. Except these actual mass, there are many right atrial structures which can mimic an abnormal mass and may not be shown well on routine standard views by echocardiography. These structures included the Eustachian

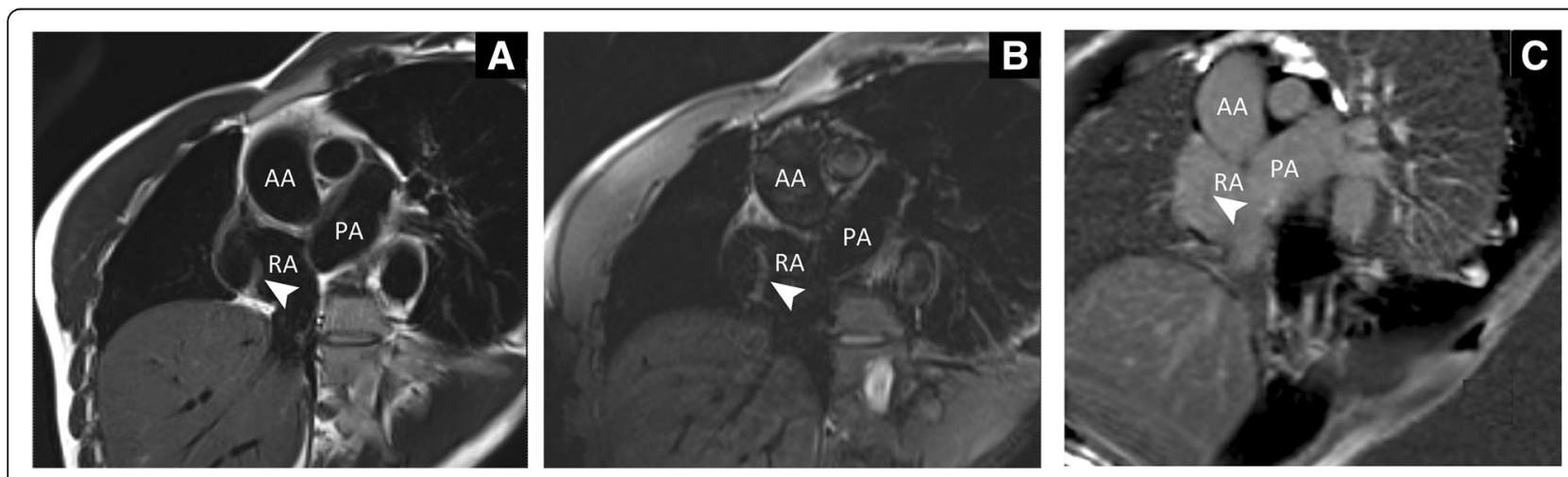

Fig. 3 Short axis view. a. On T2-STIR image, the mass (arrowhead) had similar signal intensity to myocardium revealing a myocardial structure and excluding lipoma. b. On T2-STIR with fat suppression image, the consequence is consistent with (a). c. LGE-sequences indicated no abnormal enhancement of the mass (arrowhead). T2-STIR, T2-weighted short tau inversion recovery; RA, right atrium; PA, pulmonary artery; AA, ascending aorta; LGE, late gadolinium enhancement 


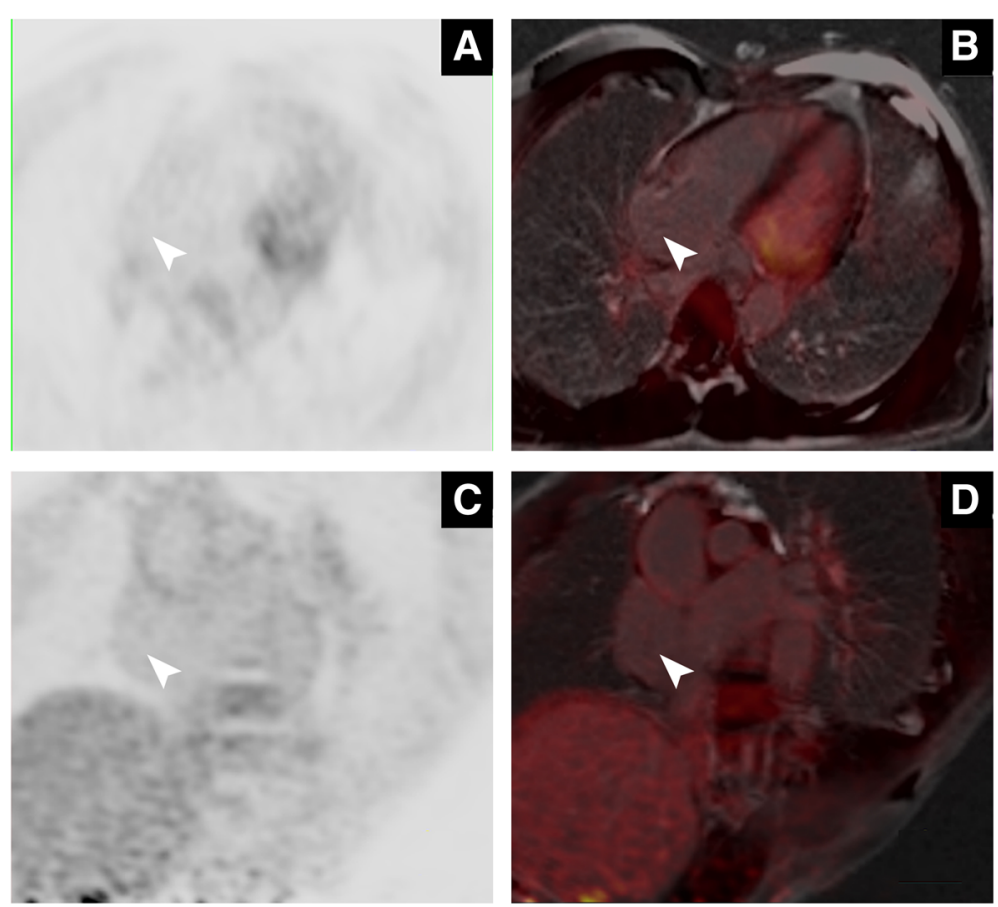

Fig. 4 The four chamber view of the heart using PET (a) and fused PET/MRI (b) demonstrated no obvious radiotracer uptake on the mass (arrowheads). Short axis view of the heart using PET (c) and fused PET/MRI (d) also indicated nothing unusual on the mass (arrowheads)

valve, thebesian valve, persistent sinus venosus, Chiari network and the crista terminalis [20]. In this case, we misdiagnose the mass as tumor or thrombus on echocardiography initially and finally diagnose it as crista terminalis by other subsequent measures. It is significant for us to take full advantage of multiple methods and make comprehensive analysis on cardial mass-like structure.

The detection of the cardial mass is mainly based on echocardiography, computed tomography (CT), and cardial MRI. Echocardiography is the first choice for the detection and evaluation of cardiac space occupying lesions [21, 22]. Among them, two-dimension can helps reveal mass location, size, mobility and differentiation from extracardiac disease. And doppler echocardiography can evaluate the hemodynamic changes caused by cardiac mass, which are generally not needed in the atrium. When the heart is examined by echocardiography, we should pay attention to direct and indirect signs. The direct signs are the abnormal echo lump in the cardiac cavity. We should know its morphology, the relationship with the heart wall, activity, hardness and echo intensity. The mass effect mainly depends on the location of the tumor. The intracavitary tumor of the right atrium mainly causes the enlargement of the right atrium, the decrease of the pulmonary blood and the dilatation of the superior vena cava, similar to the mitral valve damage or the constrictive pericarditis. The high pressure signs of pulmonary circulation, which are mainly pulmonary veins, are important indications of left atrial tumor. Evaluation with echocardiography may be limited in patients with a large body habitus. Transesophageal echocardiography, with the help of esophagus probe placed in the esophagus or stomach fundus, can scan the heart from back to front. It provide better spatial resolution, high imaging quality and can be used to differentiate non-pathologic structures from pathologic ones in right atrial structure, which contributing to diagnosis better [2,23], but restricted by its invasive and not as widely available.

In recent years, multi-slice spiral-CT and MRI has been the next strategies of choice after echocardiography, which can provide definite diagnosis evidence for cardiac tumors depending on their dynamic images, high resolution, multiple plane reconstruction and the sensitivity of calcification and fat within a mass. It is of great help to the diagnosis of the lesions property, location, the scope of the invasion and the identification of the pericardial and mediastinal tumors [24]. CT scan described the crista terminalis as a band extending obliquely across the right ventricle [25]. Cardiac MRI scan described the crista terminalis as the line of union between the RA and the right auricle, which is in signal intensity to myocardium. Gadolinium contrast material is helpful in differentiating a thrombus or crista terminalis from tumor, as the former does not show enhancement [7]. However, we can't ignore the negative impact and 
contraindication such as risks from contrast use, radiation and patients with claustrophobia, pacemaker or other metallic implants. The choice of technique to use after echocardiography still depends on the actual situation and suggestion of clinician.

PET is a positron emission tomography technology which using the radiolabeled glucose analogue ${ }^{18}$ F-FDG as a tracer is a functional image technique used to differentiate metabolic changes in normal cells and malignant cells with a carcinoma of unknown origin. Compared with normal tissues, the tumor cells can take up ${ }^{18} \mathrm{~F}$-FDG more than several times and obtain this information through PET imaging $[26,27]$. It has been a new imaging technique applied to the clinic after CT and MRI. With the development of science and technology, MRI has been combined with PET representing an exciting technological advance, which provides not only functional and metabolism images but also morphologic information especially soft tissues [28]. In this case report, there was no obvious 18F-FDG uptake about the mass on PET and PET/MRI images, by means of Biograph mMR PET-MR system, which help us exclude malignant tumor more accurately. PET/MRI as an integrated imaging technology would allow simultaneous data acquisition, resulting in combined functional and morphological images with an excellent soft tissue contrast, good spatial resolution of the anatomy and accurate temporal and spatial image fusion with a broader prospect of development. However, there are some limitations for PET scan. As for benign mass such as atrial myxoma, a usual benign tumor, PET/CT scan usually presents no obvious FDG uptake, which usually is not different to normal heart cells. PET/CT scan usually has application value in differentiating malignant and benign lesions. While echocardiography has characteristic sign in atrial myxoma. To any uncertain cardial mass, we should depend on multiple detection methods and comprehensive judgment rather than focusing on single methods.

Endomyocardial biopsy (EMB) usually plays a pivotal role in the diagnosis of some diseases, which is the "gold standard" by providing heart tissue in vivo [29]. The diagnostic level of this tool is cellular and it can provide specific etiologic information for therapy and management. In our case, by comprehensive analysis of the above results, the diagnosis is specific. The EMB can be crucial for special cases diagnosed difficultly.

In addition, there were a few case reports of the prominent crista terminalis in the literature, in retrospect, diagnosed with different tools and interestingly all of them are female (Table 1). However, the case report we presented was male and the clinical history and feature were non-specific. We should pay more attention to these case, try to summarize features and avoid misdiagnosis.

\section{Conclusion}

In summary, our case report demonstrated that the crista terminalis is an unfrequent anatomy variation and example of pseudo-mass which can be easily

Table 1 Case reports on prominent crista terminalis

\begin{tabular}{|c|c|c|c|c|c|c|c|c|}
\hline Author & Sex/Age & History & TTE & 3D-TTE & TEE & CT & MRI & PET \\
\hline D'Amato [4] & $F / 71$ & Hypertension AF & $\sqrt{ }$ & & $\sqrt{ }$ & & & \\
\hline Mckay [6] & $F / 49$ & Healthy & $\sqrt{ }$ & $\sqrt{ }$ & & & & \\
\hline Akcay [1] & $\mathrm{F} / 51$ & Dyspnea Palpitations & $\sqrt{ }$ & & $\sqrt{ }$ & & & \\
\hline Gaudio [7] & $F / 68$ & Hypertension & $\sqrt{ }$ & & & & $\sqrt{ }$ & \\
\hline Pharr [9] & $\mathrm{F} / 77$ & $\begin{array}{l}\text { COPD } \\
\text { Dyspnea }\end{array}$ & $\sqrt{ }$ & & $\sqrt{ }$ & & & \\
\hline Pharr [9] & $\mathrm{F} / 74$ & Legs edema & $\sqrt{ }$ & & $\sqrt{ }$ & & & \\
\hline Pharr [8] & $F / 58$ & Dyspnea & $\sqrt{ }$ & & $\sqrt{ }$ & & & \\
\hline Alessandro [2] & $F / 26$ & ESRD & $\sqrt{ }$ & & $\sqrt{ }$ & & $\sqrt{ }$ & \\
\hline Bannas [11] & $F / 57$ & $\begin{array}{l}\text { Hypertension } \\
\text { Dyspnea }\end{array}$ & $\sqrt{ }$ & & & & $\sqrt{ }$ & \\
\hline Jin [10] & $F / 73$ & $\begin{array}{l}\text { TS } \\
\text { Dyspnea } \\
\text { PE }\end{array}$ & $\sqrt{ }$ & & $\sqrt{ }$ & $\sqrt{ }$ & $\sqrt{ }$ & \\
\hline Massimo [5] & $F / 64$ & $\begin{array}{l}\text { Dyspnea } \\
\text { Palpitations }\end{array}$ & $\sqrt{ }$ & & & $\sqrt{ }$ & $\sqrt{ }$ & \\
\hline Salim [3] & $F / 32$ & $\begin{array}{l}\text { Dyspnea } \\
\text { Dizziness } \\
\text { AF }\end{array}$ & $\sqrt{ }$ & & & & $\sqrt{ }$ & \\
\hline Present study & $M / 54$ & Healthy & $\sqrt{ }$ & & & & $\sqrt{ }$ & $\sqrt{ }$ \\
\hline
\end{tabular}

Abbreviation: $A F$ atrial fibrillation, COPD chronic obstructive pulmonary disease, ESRD end stage renal disease, $P E$ pulmonary embolism, TS tuberculosis spondylitis 
misdiagnose for a mass. There is no doubt that the usefulness of TTE and TEE for the correct detection of prominent crista terminalis is certain. PET/MRI imaging remains a good alternative if a doubt remains after the TTE and TEE examination, which can make accurate diagnosis and avoid misdiagnosis and redundant invasive measures.

\section{Additional file}

Additional file 1: Video 1. (Four chamber view of the heart using cine MRI): Cine MRI confirmed a mass (arrow-head) attached to the posterior wall of the right atrium. (AVI $833 \mathrm{~kb}$ )

\section{Abbreviations}

AA: Ascending aorta; AF: Atrial fibrillation; COPD: Chronic obstructive pulmonary disease; CT: Computed tomography; ECG: Electrocardiogram; ESRD: End stage renal disease; LA: Left atrium; LGE: Late gadolinium enhancement; LV: Left ventricle; PA: Pulmonary artery; PE: Pulmonary embolism; PET/MRI: Positron emission tomography/magnetic resonance imaging; RA: Right atrium; RV: Right ventricle; T2-STIR: T2-weighted short tau inversion recovery; TS: Tuberculosis spondylitis

\section{Acknowledgements}

The authors very much thanks the patient, who kindly consented to the publication of our case report. And we also would like to show our thankfulness to Mr. Jiajin Liu (Department of Nuclear Medicine, General Hospital of Chinese People's Liberation Army, China) and Lijun Li (Department of Cardiology, General Hospital of Chinese People's Liberation Army, China) for kindly providing images, videos and pictures of PET/MRI.

\section{Funding}

This case presentation is supported by the National Natural Science Foundation of China (grant No. 81101468, 81571841).

\section{Availability of data and materials}

All datasets used and analyzed during this current study are included in published article and available from the corresponding author on reasonable request.

\section{Authors' contributions}

JW and $\mathrm{CL}$ wrote the manuscript. GW and XB provided the analysis of pictures and images. RZ and $\mathrm{CL}$ were involved in editing and revision of the case report. All the authors were involved in the draft, revision and approval of the final version.

Ethics approval and consent to participate Not applicable.

\section{Consent for publication}

Written informed consent was obtained from the patient for publication of this case report and any accompanying images and tables.

\section{Competing interests}

The authors declare that they have no competing interests.

\section{Publisher's Note}

Springer Nature remains neutral with regard to jurisdictional claims in published maps and institutional affiliations.

\section{Author details}

'Department of Pulmonary \& Critical Care Medicine, General Hospital of Chinese People's Liberation Army, 28th Fuxing Road, Beijing 100853, China. 2Department of Cardiology, Anshan Central Hospital, 77th Zhonghua Road (South), Anshan 114001, Liaoning Province, China. ${ }^{3}$ Department of Nuclear Medicine, General Hospital of Chinese People's Liberation Army, 28th Fuxing Road, Beijing 100853, China. ${ }^{4}$ Department of Cardiology, General Hospital of Chinese People's Liberation Army, 28th Fuxing Road, Beijing 100853, China.
Received: 1 April 2018 Accepted: 24 September 2018

Published online: 07 November 2018

\section{References}

1. Akcay M, Bilen ES, Bilge M, Durmaz T, Kurt M. Prominent crista terminalis: as an anatomic structure leading to atrial arrhythmias and mimicking right atrial mass. J Am Soc Echocardiogr. 2007:20(2):197.e199-10.

2. Alessandro S, Sherif B, Amer S, Peter L, Abdulrahman AMW. Prominent crista terminalis mimicking a right atrial mass: case report. Cardiovasc Ultrasound. 2010;8(1):47

3. Salim H, Palit A, Maher A. When is a mass not a mass? An unusual presentation of prominent crista terminalis. BMJ Case Rep. 2016. https://doi. org/10.1136/bcr-2015-211532.

4. D'Amato N, Pierfelice O, D'Agostino C. Crista terminalis bridge: a rare variant mimicking right atrial mass. Eur J Echocardiogr. 2009;10(3):444.

5. Massimo B, Diletta B. A prominent crista terminalis associated with atrial septal aneurysm that mimics right atrial mass leading to atrial arrhythmias: a case report. J Med Case Rep. 2012;6(1):403.

6. Mckay T, Thomas L. Prominent crista terminalis and Eustachian ridge in the right atrium: two dimensional (2D) and three dimensional (3D) imaging. Eur J Echocardiogr. 2007:8(4):288

7. Gaudio C, Di MS, Cera M, Nguyen BL, Pannarale G, Alessandri N. Prominent crista terminalis mimicking a right atrial mixoma: cardiac magnetic resonance aspects. Fur Rev Med Pharmacol Sci. 2004:8(4):165-8.

8. JR P, VM F. Lipomatus hypertrophy of the atrial septum and prominent crista terminalis appearing as a right atrial mass. J Am Soc Echocardiogr. 2002;15(7):753-5.

9. Pharr JR, West MB, Kusumoto FM, Figueredo VM. Prominent crista terminalis appearing as a right atrial mass on transthoracic echocardiogram. J Am Soc Echocardiogr. 2002;15(7):753-5.

10. Jin ON, Kim EJ, Sun JM, Choi EH, Jin HM, Lee HR, Yun KK, Yong HS. Prominent crista terminalis in patients with embolic events. J Cardiovasc Ultrasound. 2011;19(3):156-8.

11. Bannas P, Groth M, Lund G. Typical cardiac MRI findings of a prominent crista terminalis mimicking a right atrial mass on echocardiography. Rofo. 2012;183(08):740-1.

12. Grothues F, Smith GC, Moon JC, Bellenger NG, Collins P, Klein HU, Pennell DJ. Comparison of interstudy reproducibility of cardiovascular magnetic resonance with two-dimensional echocardiography in normal subjects and in patients with heart failure or left ventricular hypertrophy. ACC Curr J Rev. 2002;11(6):44-5.

13. Pablo $P L$, Eduardo $P$, Siqueira ME, Inés GL, Matthew $C$, Adam J, Frank M, Valentín F, Jagat $N$, Javier S. Value of CMR for the differential diagnosis of cardiac masses. J Am Coll Cardiol Img. 2014;7(9):896-905.

14. Nekolla SG, Martinez-Moeller A, Saraste A. PET and MRI in cardiac imaging: from validation studies to integrated applications. Eur J Nucl Med Mol Imaging. 2009;36(1):121-30.

15. Mirowitz SA, Gutierrez FR. Fibromuscular elements of the right atrium: pseudomass at MR imaging. Radiology. 1992;182(1):231.

16. Kalman JM, Olgin JE, Karch MR, Hamdan M, Lee RJ, Lesh MD. "Cristal tachycardias": origin of right atrial tachycardias from the crista terminalis identified by intracardiac echocardiography. J Am Coll Cardiol. 1998;31(2):451.

17. Sánchezquintana D, Anderson RH, Cabrera JA, Climent V, Martin R, Farré J, Ho SY. The terminal crest: morphological features relevant to electrophysiology. Heart. 2002;88(4):406.

18. Ragland MM, Tak T. The role of echocardiography in diagnosing spaceoccupying lesions of the heart. Clin Med Res. 2006;4(1):22.

19. Yusuf SW, Bathina JD, Qureshi S, Kaynak HE, Banchs J, Trent JC, Ravi V, Daher IN, Swafford J. Cardiac tumors in a tertiary care cancer hospital: clinical features, echocardiographic findings, treatment and outcomes. Heart Int. 2012;7(1):e4

20. Ducharme A, Tardif JC, Mercier LA, Burelle D, Rodrigues A, Petitclerc R, Pelletier $\mathrm{G}$. Remnants of the right valve of the sinus venosus presenting as a right atrial mass on transthoracic echocardiography. Can J Cardiol. 1997 13(6):573-6.

21. Zhou WW, Wang HW, Liu NN, Li JJ, Wei Y, Rui Z, Xiang LB. Diagnosis of malignancy of adult mediastinal tumors by conventional and transesophageal echocardiography. Natl Med J China. 2015;128(8):1047-51.

22. Saric M, Armour AC, Arnaout MS, Chaudhry FA, Grimm RA, Kronzon I, Landeck BF, Maganti K, Michelena HI, Tolstrup K. Guidelines for the use of 
echocardiography in the evaluation of a cardiac source of embolism. J Amer Soc Echocardiogr. 2016;29(1):1.

23. Faletra FF, Ho SY, Auricchio A. Anatomy of right atrial structures by real-time 3D transesophageal echocardiography. JACC Cardiovasc Imaging. 2010;3(9): 966-75.

24. Kim EY, Choe YH, Sung K, Park SW, Kim JH, Ko Y. Multidetector CT and MR imaging of cardiac tumors. Korean J Radiol. 2009;10(2):164.

25. Hoey E, Ganeshan A, Nader K, Randhawa K, Watkin R. Cardiac neoplasms and pseudotumors: imaging findings on multidetector $\mathrm{CT}$ angiography. Diagn Interv Radi. 2012;18(1):67-77.

26. Kaya AO, Coskun U, Unlu M, Akdemir UO, Ozdemir NY, Zengin N, Benekli M, Yildiz R, Yaman E, Ozturk B. Whole body 18F-FDG PET/CT imaging in the detection of primary tumours in patients with a metastatic carcinoma of unknown origin. Asian Pac J Cancer Preve. 2008;9(4):683.

27. Tan H, Jiang L, Gao Y, Zeng Z, Shi H. 18F-FDG PET/CT imaging in primary cardiac angiosarcoma: diagnosis and follow-up. Clin Nucl Med. 2013;38(12): $1002-5$.

28. Zaidi H, Guerra AD. An outlook on future design of hybrid PET/MRI systems. Med Phys. 2011;38(10):5667.

29. Thiene G, Bruneval P, Veinot J, Leone O. Diagnostic use of the endomyocardial biopsy: a consensus statement. Virchows Arch. 2013;463(1):1-5.

Ready to submit your research? Choose BMC and benefit from:

- fast, convenient online submission

- thorough peer review by experienced researchers in your field

- rapid publication on acceptance

- support for research data, including large and complex data types

- gold Open Access which fosters wider collaboration and increased citations

- maximum visibility for your research: over $100 \mathrm{M}$ website views per year

At $\mathrm{BMC}$, research is always in progress.

Learn more biomedcentral.com/submissions 